\title{
Modelagem computacional de um conversor de energia das ondas em energia elétrica do tipo Coluna de Água Oscilante (CAO) considerando dados de estado do mar
}

Computational modeling of an Oscillating Water Column (OWC) type wave energy converter into electrical energy considering sea state data

\author{
S. O. Sabedra ${ }^{1 *}$; P. H. Oleinik ${ }^{2}$; M. N. Gomes ${ }^{1,3}$; E. D. Santos ${ }^{1,2}$; J. A. Souza ${ }^{1,3} ;$ L. \\ A. O. Rocha ${ }^{5}$; W. C. Marques ${ }^{1,4}$; L. A. Isoldi ${ }^{1,2}$ \\ ${ }^{1}$ Programa de Pós-Graduação em Modelagem Computacional (PPGMC), Universidade Federal do Rio Grande \\ (FURG), 96.203-900, Rio Grande/RS, Brasil \\ ${ }^{2}$ Escola de Engenharia (EE), Universidade Federal do Rio Grande (FURG), 96.203-900, Rio Grande/RS, Brasil \\ ${ }^{3}$ Instituto Federal de Educação, Ciência e Tecnologia do Estado do Paraná (IFPR), 83.215-750, Paranaguá/PR, Brasil \\ ${ }^{4}$ Instituto de Matemática, Estatística e Física (IMEF), Universidade Federal do Rio Grande (FURG), 96.203-900, Rio \\ Grande/RS, Brasil
}

${ }^{5}$ Departamento de Engenharia Mecânica (DEMEC), Universidade Federal do Rio Grande do Sul (UFRGS), 90.050170, Porto Alegre/RS, Brasil

*sersana@hotmail.com

(Recebido em 03 de maio de 2017; aceito em 22 de maio de 2017)

\begin{abstract}
O presente estudo utiliza dados simulados de estado de mar, obtidos através do modelo TOMAWAC, como condição de contorno para a simulação numérica do princípio de funcionamento de um conversor de energia das ondas do mar em energia elétrica do tipo Coluna de Água Oscilante (CAO). Para isso foi considerada uma região próxima à costa de Rio Grande, RS, onde os dados de altura significativa $(H s)$ foram usados para determinar a variação de velocidade vertical $(w)$ da superfície livre do mar. Então, adotando um modelo computacional simplificado, onde somente o comportamento fluidodinâmico do conversor CAO é simulado, a velocidade vertical do estado de mar foi imposta como velocidade prescrita. Para isso, o software FLUENT, que é baseado no Método dos Volumes Finitos (MVF), foi empregado. Os resultados obtidos, através da combinação dos modelos computacionais TOMAWAC e FLUENT, mostraram a coerência e a potencialidade da metodologia proposta para a avaliação do princípio de funcionamento de dispositivos CAO.

Palavras-chave: Coluna de Água Oscilante (CAO), estado de mar, simulação numérica
\end{abstract}

The present study employs simulated sea state data, obtained through the TOMAWAC model, as boundary condition for the numerical simulation of the operating principle of the Oscillating Water Column (OWC) type wave energy converter. To do so, it was considered a region near to the Rio Grande coast, RS, where significant height $(H s)$ data were used to determine the sea surface vertical velocity $(w)$ variation. Then, by adopting a simplified computational model, where only the OWC converter fluid-dynamic behavior is simulated, the sea state vertical velocity was imposed as prescribed velocity. For this, FLUENT software, which is based on the Finite Volume Method (FVM), was adopted. The obtained results, through the combination of the TOMAWAC and FLUENT computational models, show the coherence and potentiality of the proposed methodology for the operating principle evaluation of OWC devices.

Keywords: Oscillating Water Column (OWC), sea state, numerical simulation

\section{INTRODUÇÃO}

Num momento em que o consumo de energia elétrica vem sendo discutido no mundo inteiro, devido aos problemas provenientes de sua geração, há uma grande necessidade de se desenvolver alternativas que apresentam impactos ambientais menores. Além das energias renováveis que já estão em funcionamento, existe uma aposta que tem ganho destaque, devido à sua abundância, que consiste na conversão da energia das ondas oceânicas em energia elétrica. 
Mesmo que nem sempre tenha sido vista como energia útil, sabe-se que alguns registros datados do século XVIII já mencionavam o potencial energético das ondas. Porém, somente a partir de 1960 que estudos relevantes sobre o potencial energético das ondas do mar foram realizados [1]. Atualmente, existem diversas tecnologias propostas para a conversão da energia das ondas em energia elétrica, mas nenhuma delas está consolidada, sendo necessário ainda o desenvolvimento de pesquisas nesse tema. Com relação ao local de instalação, estes dispositivos podem ser classificados como conversores costeiros (onshore), conversores próximos da costa (nearshore) e conversores afastados da costa (offshore). Já de acordo com seu princípio de funcionamento podem ser classificados como Coluna de Água Oscilante (CAO), Corpos Oscilantes e Galgamento [2].

Uma das tecnologias que tem apresentado resultados promissores é a que utiliza o conversor do tipo Coluna de Água Oscilante (CAO), pois é a mais investigada na área de geração de eletricidade a partir da energia das ondas. Vários protótipos já foram instalados e testados, como: os de eixo vertical (1985, Toftstalen - Noruega; 1990 Trivandrum - Índia), e os de eixo horizontal (1999, Pico - Portugal; 2000 Limpet - Escócia; 2005 Port Kembala - Austrália) [3].

Um conversor CAO é composto, fundamentalmente, por câmara hidropneumática, chaminé, turbina e gerador. Seu princípio de funcionamento é baseado na incidência das ondas sobre a câmara hidropneumática (parcialmente submersa), que na parte inferior possui uma abertura para o mar abaixo da superfície livre da água. Na parte superior da câmara hidropneumática, está acoplada a chaminé que em sua parte superior possui uma abertura para atmosfera, como mostra a Figura 1. De acordo com $[1,4,5]$, o processo de conversão da energia das ondas do mar em energia elétrica ocorre em duas etapas: quando a onda entra na estrutura, o ar que se encontrava dentro dela é forçado a passar por uma turbina instalada na chaminé, como consequência direta do aumento de pressão na câmara hidropneumática; e quando a onda regressa ao mar, o ar passa novamente pela turbina, desta vez no sentido inverso, dada a pressão inferior no interior da câmara hidropneumática. A rotação da turbina aciona o gerador e assim é convertida a energia das ondas em energia elétrica. O movimento da superfície livre da água no interior da câmara hidropneumática, devido à incidência das ondas, pode ser aproximado e entendido como o de um pistão, causando a compressão e a descompressão do ar (Figura 1a).

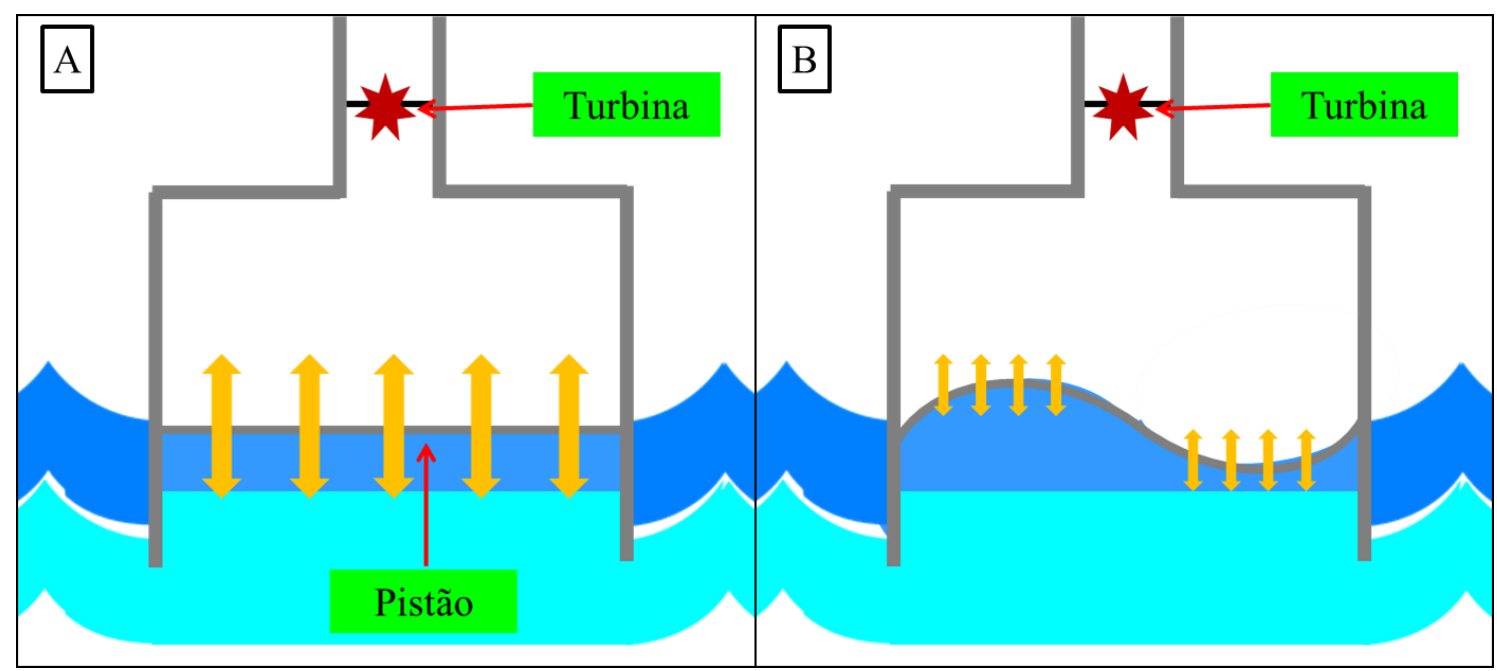

Figura 1: Representação esquemática do conversor CAO: (a) movimento idealizado de pistão e (b) movimento real causado pela superfície livre da água

Sabe-se que a modelagem computacional tem sido uma forte aliada de pesquisadores, simulando numericamente fenômenos físicos através de uma sistemática que envolve engenharia, matemática e ciência da computação. Especificamente, para a análise do princípio de funcionamento de conversores $\mathrm{CAO}$ duas abordagens computacionais merecem destaque: a Metodologia Ar e a Metodologia VOF.

A Metodologia Ar considera unicamente o escoamento de ar no interior do dispositivo sendo, portanto, uma abordagem simplificada. Emprega um domínio computacional no qual somente o 
conversor CAO é considerado. Uma condição de contorno transiente de velocidade vertical prescrita (por exemplo uma função senoidal ou cossenoidal), aplicada na parte inferior do domínio, reproduz o efeito de pistão causado pelo movimento oscilante da superfície livre da água, como mostra a Figura 1a. Essa abordagem foi usada em [3], para analisar o escoamento tridimensional do ar no interior de um conversor CAO equipado com duas chaminés verticais; e em [6], onde um estudo completo 3D de um dispositivo CAO semelhante ao da ilha do Pico em Portugal, incluindo a modelagem da turbina, foi realizado.

Já a Metodologia VOF é composta pelo método Volume of Fluid (VOF) proposto por [7] é usado, reproduzindo a interação entre a água do mar e a atmosfera. O VOF é um modelo numérico multifásico no qual dois ou mais fluidos imiscíveis interagem durante o escoamento [8]. Nessa abordagem, o domínio computacional é composto pelo conversor CAO inserido em um canal onde ondas são geradas numericamente e incidem sobre o conversor $\mathrm{CAO}$, promovendo o escoamento de ar no interior do dispositivo (Figura 2b). A Metodologia VOF foi usada, por exemplo, em $[9,10,4]$, propiciando o desenvolvimento de estudos 2D e 3D sobre o princípio de funcionamento do conversor CAO. Cabe destacar ainda que [11,2] apresentam um estudo comparativo entre as Metodologias Ar e VOF.

No presente trabalho a Metodologia Ar será empregada, considerando como condição de contorno de velocidade prescrita dados simulados de estado do mar. Para isso o software TOMAWAC foi utilizada para a geração dos dados de estado de mar enquanto os softwares GAMBIT e FLUENT foram usados, respectivamente, na construção/discretização do domínio computacional e na solução das equações de conservação.

Portanto, o objetivo deste trabalho é propor um modelo computacional que permita a simulação numérica do princípio de funcionamento de um conversor CAO a partir de um estado de mar simulado através de um modelo numérico espectral de ondas geradas pelo vento numa região localizada na costa de Rio Grande - RS.

\section{MATERIAL E MÉTODOS}

Nesta seção será apresentada a modelagem computacional, a verificação do modelo computacional e o estudo de caso utilizados neste trabalho.

\subsection{MODELAGEM COMPUTACIONAL}

De acordo com [12], o engenheiro ou projetista tem à sua disposição, fundamentalmente, três ferramentas para desenvolver um projeto ou analisar um problema: métodos analíticos; métodos numéricos (experimentação numérica); e experimentação em laboratório.

Entretanto em [13] é afirmado que a modelagem computacional permite analisar numericamente os mais diversos fenômenos reais sem a necessidade de montagem de experimentos complicados que, muitas vezes, envolvem fatores que não são importantes para o fenômeno que se deseja analisar. A simulação computacional facilita a compreensão de fenômenos físicos. Essas qualidades tornam as técnicas de simulação um instrumento indispensável para pesquisas científicas e projetos de engenharia.

O uso de técnicas numéricas para a solução de problemas complexos da engenharia e da física é uma realidade, graças ao vertiginoso desenvolvimento de computadores de alta velocidade e de grande capacidade de armazenamento. Em função dessa disponibilidade computacional, que cresce exponencialmente, o desenvolvimento de algoritmos para a solução dos mais diversos problemas tem recebido enorme atenção dos analistas numéricos e engenheiros, fazendo aumentar também em taxas acentuadas, o número de pesquisadores e usuários da simulação numérica [12].

No presente estudo, para simular numericamente o princípio de funcionamento do dispositivo CAO, uma abordagem simplificada do fenômeno físico, chamada de Metodologia Ar, será adotada. Nessa abordagem, a incidência das ondas sobre o conversor não será levada em conta. Somente o conversor CAO e o escoamento de ar em seu interior serão considerados. A variação 
da velocidade do escoamento de ar na entrada da câmara hidropneumática será definida a partir de dados simulados de estado de mar, ou seja, a elevação da superfície livre do oceano, ao longo do tempo, em uma determinada localização, será usada como condição de contorno do modelo computacional aqui apresentado. Em trabalhos anteriores, essa abordagem já foi empregada em $[3,6,11,2]$, porém a utilização de dados simulados de estado de mar é um aspecto inédito do presente estudo.

Esse modelo computacional se baseia no fato de que as ondas, ao incidirem sobre o conversor CAO, causam no interior da câmara hidropneumática uma variação na elevação da superfície livre da água. Como já foi explicada, essa variação de altura da superfície livre da coluna de água dentro do dispositivo pode ser considerada como um pistão que pressuriza e despressuriza o ar. Sendo assim, é possível representar esse movimento de pistão aplicando como condição de contorno uma variação da velocidade do ar na entrada da câmara hidropneumática do conversor CAO (ver Figura. 1a). Sendo assim, no interior do domínio computacional bidimensional mostrado na Figura. 2a, um escoamento isotérmico e incompressível de ar será numericamente simulado.
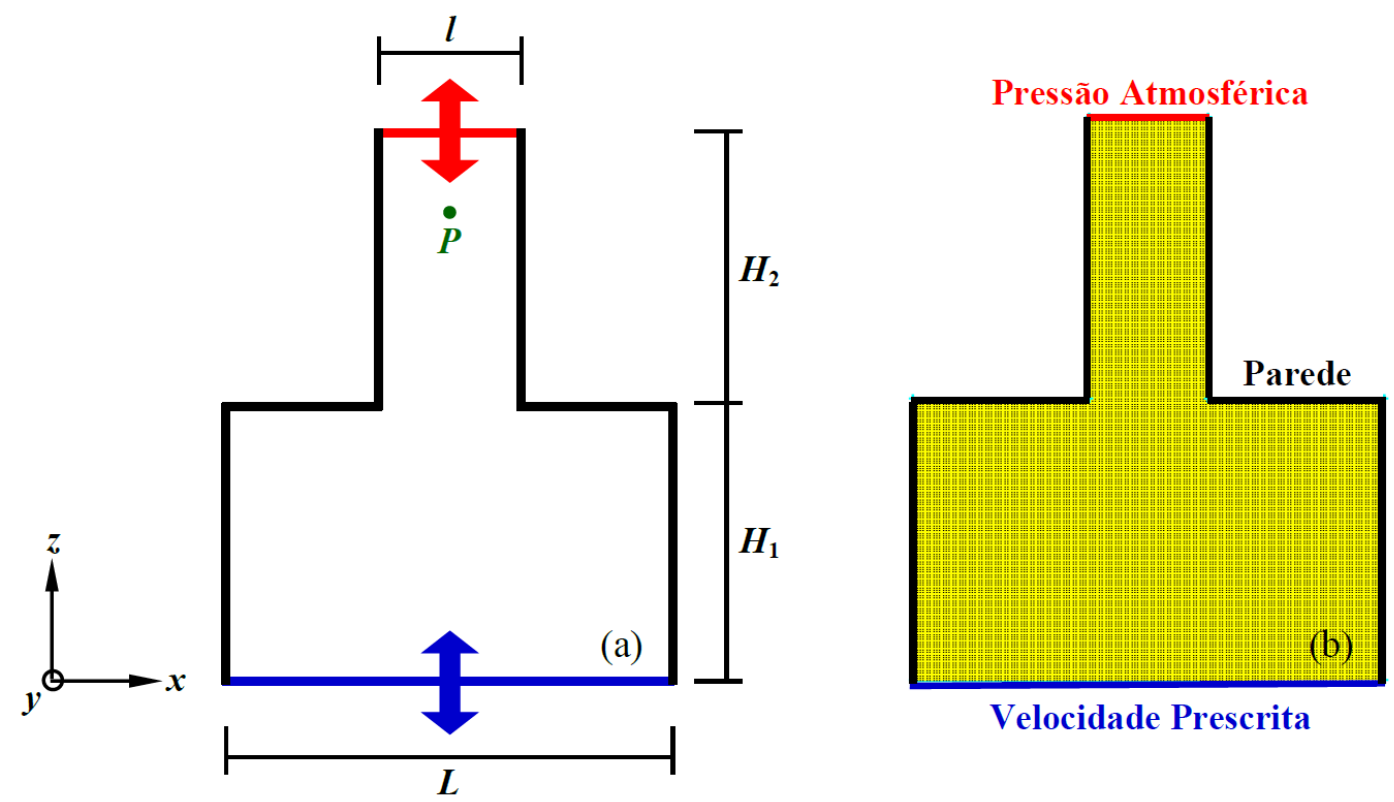

Figura 2: Domínio Computacional: (a) dimensões e (b) malha e condições de contorno

O modelo matemático é formado pelas equações de conservação da massa e da quantidade de movimento, definidas, respectivamente, por $[12,14]$ :

$\frac{\partial \rho}{\partial t}+\nabla \cdot(\rho \vec{v})=0$

$\frac{\partial}{\partial t}(\rho \vec{v})+\nabla \cdot(\rho \vec{v} \vec{v})=-\nabla p+\nabla(\mu \overline{\bar{\tau}})+\rho \vec{v}$

onde $\rho$ é a massa especifica $\left[\mathrm{kg} / \mathrm{m}^{3}\right], \vec{v}$ é o vetor velocidade do escoamento $[\mathrm{m} / \mathrm{s}], p$ é a pressão $\left[\mathrm{N} / \mathrm{m}^{2}\right], \mu$ é a viscosidade dinâmica $[\mathrm{kg} / \mathrm{m} \cdot \mathrm{s}], \overline{\bar{\tau}}$ é o tensor de tensões $\left[\mathrm{N} / \mathrm{m}^{2}\right]$ e $\vec{g}$ é a aceleração da gravidade $\left[\mathrm{m} / \mathrm{s}^{2}\right]$.

Nas simulações numéricas realizadas nesse trabalho foram adotados: o algoritmo PISO (Pressure Implicit solution by Split Operator) para o acoplamento pressão-velocidade; o método PRESTO (PREssure STaggering Option) para a discretização da pressão; e o esquema de advecção upwind de primeira ordem para os termos advectivos. Além disso o modelo de turbulência $k-\varepsilon$ foi empregado. As soluções foram consideradas convergidas para resíduos de $1 \times 10^{-5}$. 


\subsection{VERIFICAÇÃO DO MODELO COMPUTACIONAL}

De acordo com [14], o procedimento de verificação consiste em avaliar se um modelo computacional está resolvendo adequadamente as equações que governam o fenômeno físico estudado. Para isso, é necessário comparar os resultados numéricos obtidos com o modelo computacional a ser verificado com soluções analíticas ou com outras soluções numéricas.

Então, a partir do estudo de caso apresentado em [11], a verificação do modelo computacional usado no presente trabalho foi realizada. Para isso, um conversor CAO, em escala de laboratório, com $L=0.20 \mathrm{~m}, l=0.05 \mathrm{~m}, H_{1}=0.15 \mathrm{~m}$ e $H_{2}=0.15 \mathrm{~m}$ (ver Figura 2a) foi usado. O domínio computacional foi discretizado com elementos quadrilaterais de tamanho $0.001 \mathrm{~m}$, gerando uma malha regular com 37500 células computacionais (Figura 2b). Já a discretização temporal foi feita com uma passo de tempo de 0.01 [s], e a simulação numérica foi desenvolvida durante 6.00 [s]. Para representar a variação de velocidade vertical $(w)$ do ar na entrada da câmara hidropneumática, como condição de contorno, foi considerada a seguinte função transiente [6]:

$w(t)=\frac{H \pi}{T} \cos \left(\frac{2 \pi t}{T}\right)$

sendo $w$ a velocidade na direção vertical [m/s], $H$ a amplitude (de $0.14 \mathrm{~m}), T$ o período (de $0.81 \mathrm{~s}$ ) e $t$ o tempo [s]. Porém, cabe destacar que, diferentemente de [11] que impôs essa condição de contorno através de uma UDF (User Defined Function) no FLUENT, no presente trabalho valores discretos de tempo e velocidade obtidos a partir da Equação 2, a cada $0.01 \mathrm{~s}$, foram impostos como condição de contorno através da ferramenta table data do software FLUENT.

Para comparar os resultados obtidos com o modelo computacional proposto com os de [11], foi monitorada a velocidade $w$ (na direção $z$ ) do escoamento do ar, ao longo do tempo, em um ponto $P$ localizado na chaminé do conversor CAO, em $x=0.10 \mathrm{~m}$ e $z=0.20 \mathrm{~m}$ (ver Figura 2a). No presente estudo $w$ foi registrada a cada $0.01 \mathrm{~s}$.

Com isso, na Figura 3 é possível observar os resultados obtidos com o modelo numérico proposto em comparação com os resultados obtidos em [11]. Conforme já foi mencionado anteriormente, duas metodologias foram empregadas em [11]: Metodologia Ar que, com exceção da maneira de imposição da velocidade prescrita é, fundamentalmente, o mesmo modelo computacional proposto aqui; e a Metodologia VOF, onde as ondas geradas numericamente através do modelo VOF incidem sobre o conversor CAO. Por isso, para que fosse possível uma comparação entre essas três metodologias, a Figura 3 compara os resultados obtidos durante um intervalo de tempo compreendido entre $4.70 \mathrm{~s}$ e $5.50 \mathrm{~s}$.

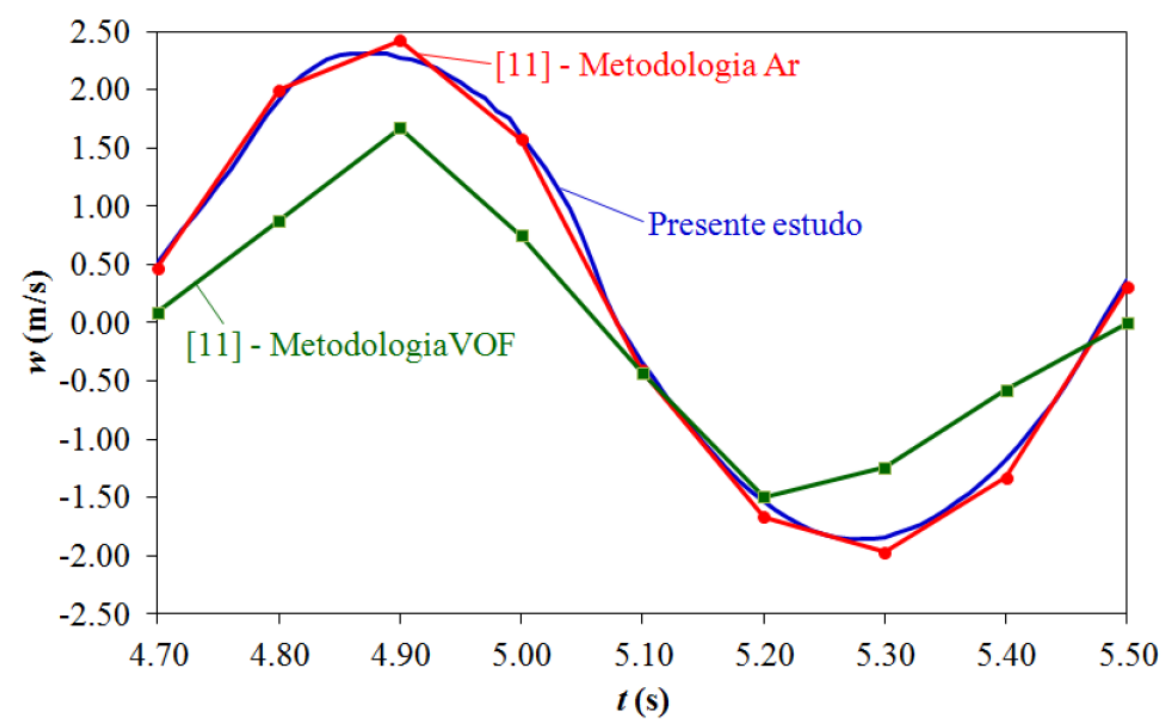

Figura 3: Comparação da variação de velocidade vertical na chaminé do conversor CAO 
É possível notar na Figura 3 que todas as soluções numéricas apresentam a mesma tendência. Além disso, fica evidente que os resultados do presente estudo estão em concordância com os de [11] gerados com a Metodologia Ar, verificando assim a imposição da condição de contorno de velocidade através da ferramenta table data. Também, como já era esperado, existe uma diferença em relação à Metodologia VOF, porém os resultados do presente estudo acompanham a mesma tendência de variação da velocidade e são ligeiramente mais próximos do que os gerados em [11] com a Metodologia Ar.

Cabe destacar que o motivo que justifica essa abordagem simplificada através da Metodologia $\mathrm{Ar}$, onde somente o conversor CAO e o escoamento de ar em seu interior são considerados, é a significativa redução no tempo de processamento. Para o caso da Figura 3, com a Metodologia VOF é necessário um tempo de processamento de aproximadamente $8 \mathrm{~h}(28800 \mathrm{~s})$, já com a Metodologia Ar o tempo de processamento é em torno de $0.5 \mathrm{~h}$ (1800 s). Essa diferença no tempo de processamento ocorre devido à redução do domínio computacional e à simplificação no equacionamento promovido pela Metodologia Ar quando comparada à Metodologia VOF.

\subsection{ESTUDO DE CASO}

A partir da verificação do modelo computacional proposto, um estudo de caso foi desenvolvido considerando dados simulados de estado de mar, obtidos através do modelo TOMAWAC.

O TOMAWAC, componente do sistema de modelagem TELEMAC (www.opentelemac.org) é um modelo de ondas de terceira geração que calcula o estado de mar resolvendo a equação de conservação de densidade de ação para o espectro direcional das ondas, dada por:

$$
\frac{\partial N(f, \theta)}{\partial t}+\frac{\partial \dot{x} N}{\partial x}+\frac{\partial \dot{y} N}{\partial y}+\frac{\partial \dot{k}_{x}}{\partial k_{x}}+\frac{\partial \dot{k}_{y}}{\partial k_{y}}=Q\left(k_{x}, k_{y}, x, y, t\right)
$$

onde $N$ é o espectro direcional das ondas, $x$ e $y$ são o sistema de coordenadas, $k x$ e $k y$ são, respectivamente, as componentes em $x$ e $y$ do vetor número de onda, $Q$ é o termo fonte e $t$ o tempo. A Equação (4) expressa que, em uma situação geral de ondas se propagando em um meio não homogêneo e instável, a densidade de ação da onda é conservada dentro dos termos fonte e sumidouro, definidos por $Q$.

O TOMAWAC calcula ondas de gravidade levando em consideração a maioria dos processos físicos envolvidos, como empolamento, arrebentação parcial, dissipação devida à fricção com o fundo, interações não lineares entre ondas e refração induzida pela batimetria. Por outro lado, o TOMAWAC não leva em consideração os efeitos de difração e reflexão.

O TOMAWAC foi previamente calibrado para a região de estudo (Fig. 4a) em [15]. Para obter as séries temporais de período médio $(T m)$ e de altura significativa $(H s)$ para o estado de mar, respectivamente Figuras. $4 \mathrm{~b}$ e $4 \mathrm{c}$, utilizadas neste artigo, o TOMAWAC foi inicializado do repouso. Os contornos oceânicos da região simulada (Fig. 4a) foram forçados pela imposição de altura significativa, período de pico e direção de pico, baixados do banco de dados gerado pelo modelo de previsão WAVEWATCHIII (ftp://polar.ncep.noaa.gov/history/waves). A fronteira superficial foi forçada por ventos da NOAA (National Oceanic and Atmospheric Administration) do Projeto Reanalysis NCEP/NCAR (www.esrl.noaa.gov/psd/data/gridded/data.ncep.reanalysis. html).

O domínio computacional simulado com o TOMAWAC (Figura 4a) cobre a região da plataforma continental sul sudeste do Brasil. Nele, foi escolhida uma localização próxima à costa de Rio Grande, RS, representada pelo ponto 1 na Figura 4a, situada a $32^{\circ} 27^{\prime} 35^{\prime \prime}$ de latitude e $52^{\circ} 05^{\prime} 01 "$ de longitude, possuindo uma profundidade de 19.97 [m]. Já o período médio $(\mathrm{Tm})$, Figura $4 \mathrm{~b}$, e a altura significativa $(H s)$, Figura $4 \mathrm{c}$, do estado de mar no ponto 1 foram monitorados, a cada $6 \mathrm{~h}(21600 \mathrm{~s})$, durante todo o ano de 2013 e janeiro de 2014, totalizando 396 dias. Assim, a partir da variação da altura significativa ao longo do tempo foi possível determinar a variação de velocidade vertical $(w)$ da superfície livre do mar, conforme mostra a Figura 4d. 

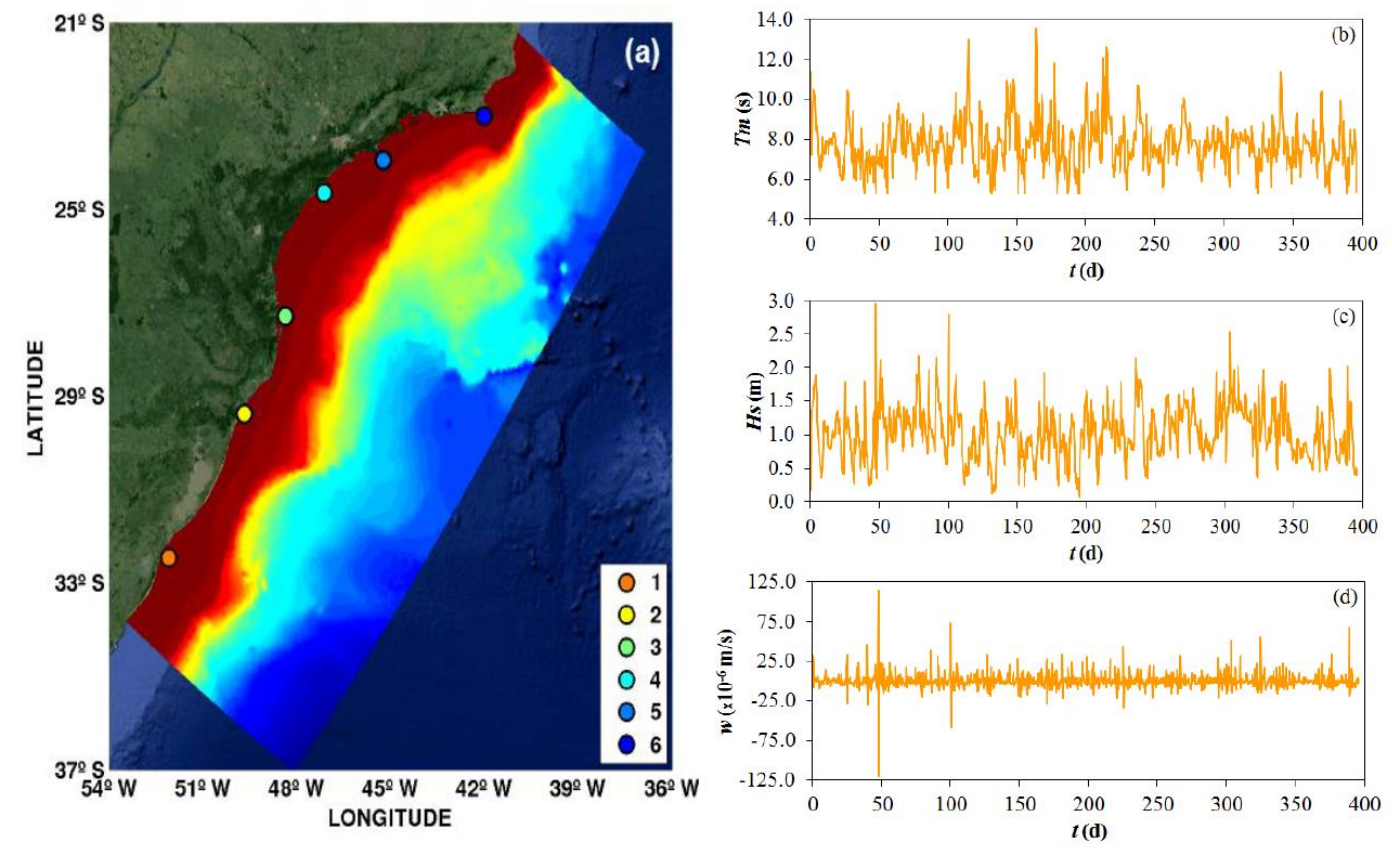

Figura 4: Ponto 1: (a) Representação geográfica, (b) período médio, (c) altura significativa e (d) velocidade vertical

\section{RESULTADOS E DISCUSSÃO}

Com base nesses dados obidos com o modelo TOMAWAC foi possível simular numericamente o princípio de funcionamento de um conversor CAO. Para a definição da geometria e dimensões do dispositivo foi considerada a recomendação teórica apresentada em [4]. A partir do valor médio dos períodos da Figura $4 \mathrm{~b}$, de 7.74 [s], as dimensões adotadas para o conversor CAO são: $L=31.56 \mathrm{~m}, l=3.18 \mathrm{~m}, H_{1}=2.25 \mathrm{~m}$ e $H_{2}=9.55 \mathrm{~m}$ (ver Figura $2 \mathrm{a}$ ), que obedecem a indicação de $0.03<H 1 / L<0.10$ feita para ondas incidentes com $T=8$ [s] em [4]. $\mathrm{O}$ domínio computacional foi então discretizado com quadriláteros de comprimento máximo de 0.1 m, formando uma malha com 10340 células (Figura 5).

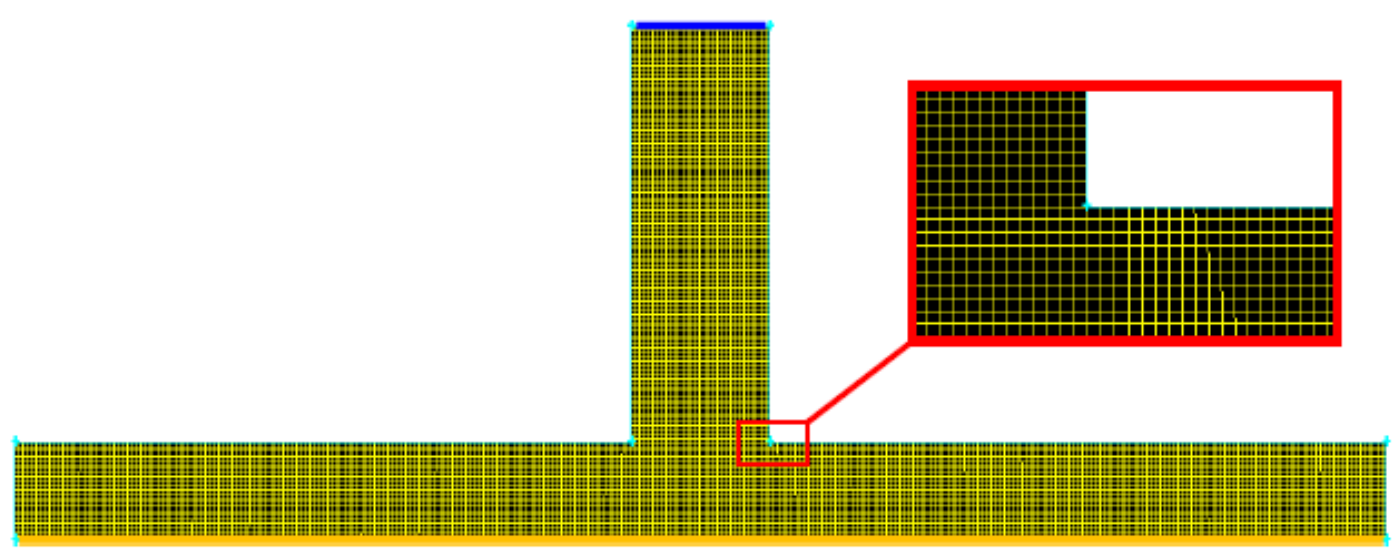

Figura 5: Discretização do domínio computacional usado no estudo de caso

A variação de velocidade da superfície livre do mar (Figura 4d) foi imposta como condição de contorno de velocidade vertical prescrita na entrada da câmara hidropneumática do conversor CAO (linha laranja na Figura 5). Um passo de tempo de 21600 [s] foi usado para a discretização temporal. Os demais procedimentos numéricos são iguais aos anteriormente descritos e usados na verificação do modelo computacional. 
Durante a simulação duas sondas numéricas foram usadas: uma linha na entrada da câmara hidropneumática (linha laranja na Figura 5) e uma linha na saída da chaminé (linha azul na Figura 5). Nelas foram monitoradas a velocidade vertical média do escoamento de ar. Na entrada do dispositivo a velocidade é prescrita, servindo a sonda numérica somente para mostrar que a variação de velocidade da Figura 4d foi realmente imposta ao modelo computacional. Já a sonda numérica na saída da chaminé tem como objetivo mostrar a diferença de magnitude na velocidade vertical do escoamento de ar, causada pelo conversor CAO. Nas Figuras. 6a e 6b são apresentadas a variação transiente da velocidade vertical do ar, respectivamente, na entrada da câmara e na saída da chaminé, obtidas numericamente.

É possível observar, comparando as Figuras $6 \mathrm{a}$ e $6 \mathrm{~b}$, que um aumento significativo na magnitude da velocidade vertical no escoamento acontece. Esse comportamento já era esperado, entretanto cabe ressaltar que para o ponto analisado (ver Figura 4a), não é de conhecimento dos autores que estudo semelhante tenha sido desenvolvido. Observa-se também que existe uma coerência entre o comportamento da velocidade vertical do ar na entrada (Figura 6a) e na saída (Figura 6b) do dispositivo. Esse fato fica evidente, por exemplo, nos dias 49 e 51, onde picos de velocidade na entrada causaram picos de velocidade na saída do conversor CAO.

Além disso, cabe destacar que, as Figuras 6a e 4d apresentam a mesma variação de velocidade ao longo do tempo (porém estão plotadas em escalas de velocidade diferentes). Isso comprova, mais uma vez, que a imposição de velocidade prescrita usando a ferramenta table data do FLUENT pode ser empregada para simulações numéricas do princípio de funcionamento de conversores do tipo CAO.

Por fim, cabe ressaltar que a Metodologia Ar, por se tratar de uma abordagem simplificada, não permite avaliar o fenômeno da amplificação que ocorre nos conversores $\mathrm{CAO}$. $\mathrm{O}$ fator de amplificação de um conversor CAO é definido como a relação entre a elevação da coluna de água dentro da câmara hidropneumática e a elevação da onda incidente [4]. Portanto, como no presente trabalho a elevação do estado de mar é diretamente considerada para a aplicação da condição de contorno de velocidade prescrita no modelo computacional, o fator de amplificação é igual a 1.
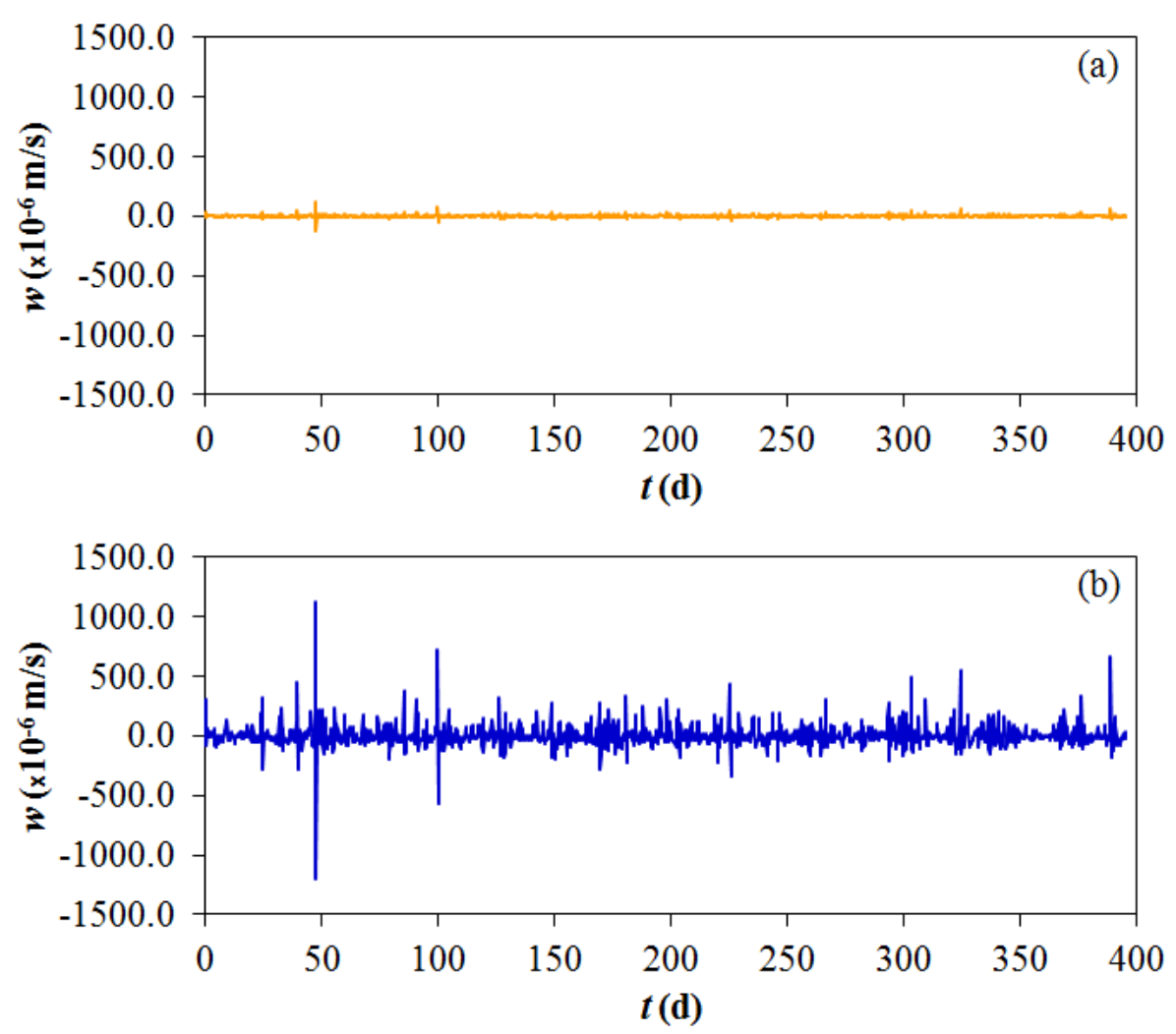

Figura 6: Variação da velocidade vertical do escoamento de ar no dispositivo CAO: (a) na entrada e (b) na saída 


\section{CONCLUSÃO}

O trabalho aqui apresentado propôs um modelo computacional para a análise do princípio de funcionamento de conversores $\mathrm{CAO}$, usado na conversão da energia das ondas do mar em energia elétrica. Esse modelo computacional baseia-se na Metodologia Ar, anteriormente aplicada por [3, 6,11 e 2] em suas pesquisas. Essa abordagem simplificada considera somente o dispositivo CAO e o escoamento de ar em seu interior. Entretanto, na modelagem computacional proposta aqui, a imposição da variação transiente de velocidade vertical do ar na entrada da câmara hidropneumática é feita a partir de dados simulados de estado de mar, sendo este um aspecto inédito da presente pesquisa.

Os resultados obtidos mostram a capacidade do modelo computacional em simular numericamente o comportamento fluidodinâmico do escoamento de ar no conversor CAO. Isso é comprovado pelo significativo aumento da magnitude da velocidade, quando se compara o escoamento na entrada (Figura 6a) e na saída (Figura 6b) do dispositivo. Além disso, observou-se também uma coerência entre a variação de velocidade imposta como condição de contorno e a variação de velocidade obtida como resposta do modelo na saída do conversor CAO. Porém, por ser uma abordagem simplificada, onde não é considerada a incidência das ondas sobre o conversor $\mathrm{CAO}$, o fenômeno da amplificação não pode ser avaliado, sendo o fator de amplificação sempre igual a 1 .

Cabe ressaltar ainda que, com a modelagem computacional proposta, foi necessário um tempo de processamento de aproximadamente $30 \mathrm{~h}(1.25 \mathrm{~d})$ para simular numericamente um intervalo de tempo de 9504 h (396 d) de um estado de mar simulado. Em outros trabalhos, desenvolvidos pelo nosso grupo de pesquisa, que utilizam a Metodologia VOF (onde o escoamento de ar no interior do conversor $\mathrm{CAO}$ ocorre devido à incidência de ondas sobre o dispositivo), quando ondas regulares são consideradas (o que também é uma simplificação em relação ao estado de mar), um tempo de processamento de $4 \mathrm{~h}$ permite a simulação numérica de um intervalo de apenas $30 \mathrm{~s}$; já quando um espectro de ondas é considerado, são necessárias $48 \mathrm{~h}$ de processamento para a simulação numérica de um intervalo de somente $100 \mathrm{~s}$. Sendo assim, conclui-se que a modelagem computacional proposta aqui, apesar de não considerar a incidência das ondas sobre o conversor $\mathrm{CAO}$, se justifica pela redução significativa no tempo de processamento e pelo uso de condições de contorno de um estado de mar real.

Por fim, é importante ressaltar que o potencial mostrado pelo modelo computacional apresentado nesse estudo preliminar indica que, em trabalhos futuros, testes de independência de malha e de passo de tempo devem ser desenvolvidos, visando melhorar suas condições de processamento. Além disso, pretende-se usar o modelo proposto aqui, também, em simulações numéricas tridimensionais e em estudos de otimização da geometria de conversores $\mathrm{CAO}$, não somente para o ponto 1 da Figura 4a mas também para outros pontos ao longo da costa brasileira.

\section{AGRADECIMENTOS}

Os autores agradecem à FURG, à CAPES e ao CNPq pelo apoio.

\section{REFERÊNCIAS BIBLIOGRÁFICAS}

1. Cruz JMBP, Sarmento AJNA. Energia das ondas. Instituto do Ambiente, Alfragide, 2004.

2. Gomes M das N. Modelagem computacional de um dispositivo coluna d'água oscilante de conversão de energia das ondas do mar em energia elétrica [dissertação]. Rio Grande (RS): Universidade Federal do Rio Grande (FURG); 2010. 209 p.

3. Conde JMP, Gato LMC. Numerical study of the air-flow in an oscillating water column wave energy converter. Renewable Energy. 2008; 33:2637-2644. doi: 10.1016/j.renene.2008.02.028.

4. Gomes $\mathrm{M}$ das $\mathrm{N}$. Constructal design de dispositivos conversores de energia das ondas do mar em energia elétrica do tipo coluna de água oscilante [tese]. Rio Grande (RS): Universidade Federal do Rio Grande (FURG); 2014. 168 p.

5. Falcão AFO, Henriques JCC. Oscillating-water-column wave energy converters and air turbines: A review. Renewable Energy. 2016; 85:1391-1424. doi:10.1016/j.renene.2015.07.086. 
6. Marjani A, Ruiz FC, Rodriguez MA, Santos MTP. Numerical modelling in wave energy conversion systems. Energy. 2008; 33:1246-1256.

7. Hirt CW, Nichols BD. Volume of fluid (VOF) method for the dynamics of free boundaries. Journal of Computational Physics. 1981; 39(1):201-225.

8. FLUENT (version 6.3.16), 2006, User Manual, ANSYS Inc.

9. Horko M. CFD Optimizations of an Oscillating Water Column Energy converter, [dissertação]. The University of Western Australia; 2007. 145 p.

10. Dos Santos ED, Machado BN, Lopes N, Souza JA, Texeira PRF, Gomes MN, Isoldi LA, Rocha LAO, 2013, Constructal design of wave energy converters. In: Constructal Law and the Unifying Principle of Design - Rocha LAO, Lorente S, Bejan A (Org.). Understanding Complex Systems. 1ed; 2013; New York, Springer (NY), vol. 1; p. 275-294.

11. Gomes M das N, Olinto CR, Isoldi LA, Souza JA, Rocha LAO. Computational modeling of the air-flow in na oscillating water column system. In: Proceedings of 20th International Congress of Mechanical Engineering (COBEM); 2009, Gramado (RS). p. 1-10.

12. Maliska C. Transferência de calor e mecânica dos fluidos computacional. Livros Técnicos e Científicos Editora S.A., Rio de Janeiro; 2004. 453 p.

13. Andrade DC, Germano JSE. Visualizador 3D para aplicações no ensino de ciências básica. In: Congresso Brasileiro de Ensino de Engenharia; 2003; Rio de Janeiro (RJ). vol. 1; p. 1-10.

14. Versteeg HK, Malalasekera W. An Introduction to Computational Fluid Dynamics - The Finite Volume Method. Pearson, Inglaterra; 2007. 503 p.

15. Oleinik PH, Marques WC, Kirinus EP. Estimate of the Wave Climate on the Most Energetic Locations of the South-Southeastern Brazilian Shelf. Defect and Diffusion Forum. 2017;370:130-140. 